This item was submitted to Loughborough's Research Repository by the author.

Items in Figshare are protected by copyright, with all rights reserved, unless otherwise indicated.

\title{
Angle-resolved photoemission in doped charge-transfer Mott insulators
}

PLEASE CITE THE PUBLISHED VERSION

LICENCE

CC BY-NC-ND 4.0

REPOSITORY RECORD

Alexandrov, A.S., and C.J. Dent. 2019. "Angle-resolved Photoemission in Doped Charge-transfer Mott Insulators". figshare. https://hdl.handle.net/2134/1204. 


\title{
Angle-resolved photoemission in doped charge-transfer Mott insulators
}

\author{
A.S. Alexandrov and C. J. Dent \\ Department of Physics, Loughborough University, Loughborough LE11 3TU, U.K.
}

\begin{abstract}
A theory of angle-resolved photoemission (ARPES) in doped cuprates and other charge-transfer Mott insulators is developed taking into account the realistic $(\mathrm{LDA}+\mathrm{U})$ band structure, (bi)polaron formation due to the strong electronphonon interaction, and a random field potential. In most of these materials the first band to be doped is the oxygen band inside the Mott-Hubbard gap. We derive the coherent part of the ARPES spectra with the oxygen hole spectral function calculated in the non-crossing (ladder) approximation and with the exact spectral function of a one-dimensional hole in a random potential. Some unusual features of ARPES including the polarisation dependence and spectral shape in $\mathrm{YBa}_{2} \mathrm{Cu}_{3} \mathrm{O}_{7}$ and $\mathrm{YBa}_{2} \mathrm{Cu}_{4} \mathrm{O}_{8}$ are described without any Fermi-surface, large or small. The theory is compatible with the doping dependence of kinetic and thermodynamic properties of cuprates as well as with the d-wave symmetry of the superconducting order parameter.
\end{abstract}

PACS numbers:74.20.Mn,74.20.-z,74.25.Jb

\section{Introduction}

The concept of polarons led to the discovery of the copper oxide superconductors. The expectation was that if 'an electron and a surrounding lattice distortion with a high effective mass can travel through the lattice as a whole, and a strong electron-phonon coupling exists, the perovskite insulator cauld be turned into a high temperature superconductor' 1 . In this paper we develop the theory of ARPES in doped charge-transfer Mott insulators based on the bipolaron theory 3 which describes some unusual ARPES features of high- $\mathrm{T}_{c} \mathrm{YBa}_{2} \mathrm{Cu}_{3} \mathrm{O}_{7-\delta}$ (Y123), $\mathrm{YBa}_{2} \mathrm{Cu}_{4} \mathrm{O}_{8}$ (Y124) and a few other materials,

ARPES data in cuprates remains highly controversiall One of the surprising features is a large Fermi surface claimed to exist in a wide range of doping fitting well the LDA band structures in the earlier studies. This should evolve with doping as $(1-x)$ in a clear contradiction with low frequency kinetics and thermodynamics (see, for example 10 , which show an evolution proportional to $x$ in wide range of doping including the overdoped region 11 ( $x$ is the number of holes introduced by doping). Now it is established, however, that there is a normal state (pseudo)rap in ARPES and tunnelling, existing well above $\mathrm{T}_{c}$ 2] 13 , so that some segments of a "large Fermi surface' are actually missing 44 . The temperature and doping dependence of the gap still remain a subject of coptroversy. While kinetid 9 , thermodynamic 10 , tunnelling 13 and some ARPES14.15 data suggest that the gap opens at any relevant temperature in a wide range of doping, other ARPES studiest 19 claim that it exists only in underdoped samples below a characteristic temperature $\mathrm{T}^{*}$.

Perhaps the most intriguing feature of ARPES is the extremely narrow and intense peak lying below the Fermi energy, which is most clearly seen near the $\mathrm{Y}$ and $\mathrm{X}$ points in $\mathrm{Y} 124 \mathrm{~d}, \mathrm{Y} 12321$ and more recently in $\mathrm{La}_{2-x} \mathrm{Sr}_{x} \mathrm{CuO}_{4} 2$. Its angular dependence and spectral shape as well as the origin of the featureless (but dispersive) background remain unclear. Some authors 20 refer to the peak as an extended van Hove singularity (evHs) arising from the plane $\left(\mathrm{CuO}_{2}\right)$ strongly correlated band. They also implicate the resulting (quasi)1D density of states singularity as a possible origin for the high transition temperature. However, recent polarised ARPES studies of untwinned Y123 crystals of exceptional qualitye1 unambiguously refer the peak to as a narrow resonance arising primarily from the quasi-1D $\mathrm{CuO}_{3}$ chains in the buffer layers rather than from the planes. Also, careful analysis of the Eliashberg equations shows that the van Hove singularity can hardly be the origin of high $\mathrm{T}_{c}$, in sharp contrast with a naive weak-coupling estimate. Interestingly a very similar narrow peak was observed by Park et a 23 in high-resolution ARPES near the gap edge of the cubic semiconductor $\mathrm{FeSi}$ with no Fermi surface at all.

In this paper we take the view that cuprates and many other transition metal compounds are charge transfer Mott-Hubbard insulators at any level of doping24. This means that the first band to be doped is the oxygen band lying within the Hubbard gap, Fig.1. Strong coupling with high frequency phonons, unambiguously established for many oxides 25 , leads to the high-energy spectral features of an oxygen hole in an energy window about twice the Franck-Condon (pglaronic) level shift, $E_{p}$, and to the band-narrowing effect 2 . On the other hand, the low energy spectral function is influenced by the low frequency thermal lattice, spin and random fluctuations. The latter can be described as 'Gaussian white noise'. The $p$ hole polaron in oxides is almost one-dimensional due to a large difference in the $p p \sigma$ and $p p \pi$ hopping integrals and effective 'one-dimensional' localisitaion by the random potential as described in Ref.26. This allows us to explain the narrow peak in the ARPES spectra with the spectral density $A(k, E)$ of a one-fimensional particle in a Gaussian white noise potential27.

The electron-phonon interaction also binds holes into inter-site oxygen bipolarons the size of a lattice constant 28. The bipolaron density remains relatively low (below 0.15 per cell) at any relevant level of doping. The residual repulsive (Coulomb) polaron-bipolaron interaction $\mathrm{B}$ is strongly suppressed by the lattice polarisation owing to a large static dielectric constant. As 
a result, the only role of hole bipolarons in ARPES is to pin the chemical potential inside the charge transfer gap, half the bipolaron binding energy above the oxygen band edge, Fig.1a. This binding energy as well as the singlet-triplet bipolaron exchange energy are thought to be the origin of the nprmal state pseudogaps, as first proposed by one of us2. In particular, the non-Korringa temperature dependence of NMR29, and more recently the values and universal scaling with temperature of the uniform magnetic susceptibility 30,6,31 and electronic specific heat 31 were quantitatively described with bipolarons. In overdoped samples the bipolaron and polaron bands might overlap because the bipolaron binding energy becomes small30, so the chemical potential might enter the oxygen band, Fig.1b, and then a Fermi-level crossing might be seen in ARPES. The featureless background is explained as the phonon cloud of a small hole polaron, which spreads over a wide energy interval about $2 E_{p} \simeq 1 \mathrm{eV}$.

The same approach has been applied to the tunnelling spectra 32 . As a result, the temperature independent gap and the anomalous gap $/ T_{c}$ ratio, injection/emission asymmetry both in magnitude and shape, zero-bias conductance at zero temperature, the spectral shape inside and outside the gap region, temperature/doping dependence and dip-hump structure of the tunnelling conductance were described. The approach is compatible with the recent ' $\mathrm{I} D \mathrm{DA}+\mathrm{U}$ ' band structures in cuprates and manganites33, which suggest the single-particle density of states shown in Fig.1. It is clearly compatible with the doping evolution of thermodynamic and kinetic properties because holes introduced by doping into the oxygen band are the only carriers in our theory. Moreover, the derived bipolaron energy dispersion with minima at the Brillouin zone boundaries preyides $d$-wave symmetry of the Bose-Einstein condensate 32 in agreement with phasesensitive experiments34. It also provides a parameter-free expression for $T_{c}$ ip f few dozen cuprates, irrespective of the level of doping 35.36 .

Here we first derive the polaronic ARPES theory in Section 2. Then, in Section 3 we apply the simplest noncrossing approximation for the self-energy and compare it with the exact result in Section 4. The experimental ARPES results, in particular the angular dispersion, spectral shape and polarisation dependence are compared with the theory in Section 5. We conclude that the narrow peak in ARPES is an intrinsic polaron (oxygen) band found below the chemical potential by half of the bipolaron binding energy. We also arrive at the conclusion that the present experimental photoemission spectra can be understood with a small or even without any Fermi surface (depending on doping).

\section{Polaronic ARPES}

The interaction of the crystal with the electromagnetic field of frequency $\nu$ is described by the Hamiltonian (in the dipole approximation)

$$
H_{i n t}=(8 \pi I)^{1 / 2} \sin (\nu t) \sum_{\mathbf{k}, \mathbf{k}^{\prime}}\left(\mathbf{e} \cdot \mathbf{d}_{\mathbf{k}, \mathbf{k}^{\prime}}\right) c_{\mathbf{k}}^{\dagger} h_{\mathbf{k}^{\prime}}^{\dagger}+H . c .
$$

where $I$ is the intensity of the radiation with the polarisation e (we take $c=\hbar=k_{B}=1$ ), $\mathbf{k}$ is the momentum of the final state (i.e. of the photoelectron registered by the detector), $\mathbf{k}^{\prime}$ is the (quasi)momentum of the hole remaining in the sample after the emission, and $c_{\mathbf{k}}^{\dagger}$ and $h_{\mathbf{k}^{\prime}}^{\dagger}$ are their creation operators, respectively. For simplicity we suppress the band index in $h_{\mathbf{k}^{\prime}}^{\dagger}$. Due to the translational symmetry of the Bloch states, $\mid \mathbf{k}^{\prime}>\equiv u_{-\mathbf{k}^{\prime}}(\mathbf{r}) \exp \left(-i \mathbf{k}^{\prime} \cdot \mathbf{r}\right)$, there is a momentum conservation rule in the dipole matrix element,

$$
\mathbf{d}_{\mathbf{k}, \mathbf{k}^{\prime}}=\mathbf{d}(\mathbf{k}) \delta_{\mathbf{k}+\mathbf{k}^{\prime}, \mathbf{G}}
$$

with

$$
\mathbf{d}(\mathbf{k})=i e\left(N / v_{0}\right)^{1 / 2} \nabla_{\mathbf{k}} \int_{v_{0}} e^{-i \mathbf{G} \cdot \mathbf{r}} u_{\mathbf{k}-\mathbf{G}}(\mathbf{r}) d \mathbf{r},
$$

where $\mathbf{G}$ is a reciprocal lattice vector, $N$ the number of unit cells in the crystal of the volume $N v_{0}$.

The Fermi Golden Rule gives the photocurrent to be

$$
\begin{gathered}
I(\mathbf{k}, E)=4 \pi^{2} I|\mathbf{e} \cdot \mathbf{d}(\mathbf{k})|^{2} \\
\times \sum_{i, f} e^{\Omega+\mu N_{i}-E_{i}}|<f| h_{\mathbf{k}-\mathbf{G}}^{\dagger}|i>|^{2} \delta\left(E+E_{f}-E_{i}\right)
\end{gathered}
$$

where $E$ is the binding energy, $E_{i, f}$ is the energy of the initial and final states of the crystal, and $\Omega, \mu, N_{i}$ are the thermodynamic and chemical potentials and the number of holes, respectively. By definition the sum in Eq.(4) is $n(E) A(\mathbf{k}-\mathbf{G},-E)$ where the spectral function $A(\mathbf{k}-\mathbf{G}, E)=(-1 / \pi) \Im G^{R}(\mathbf{k}-\mathbf{G}, E)$ is proportional to the imaginary part of the retarded Green function (GF), and $n(E)=[\exp (E / T)+1]^{-1}$, the Fermi distribution. In the following we consider temperatures well below the experimental energy resolution, so that $n(E)=1$ if $E$ is negative and zero otherwise, and, for convenience, we put $\mathbf{G}=0$.

The spectral function depends on essential interactions of a single bole with the rest of the system. As we argued earlier 26 the most important interaction in oxides is the Fröhlich long-range electron-phonon interaction of the oxygen hole with the c-axis polarised high-frequency phonons. The Fröhlich interaction is integrafed out with the Lang-Firsov canonical transfprmation 37 . With the Quantum-Monte-Carlo technique 38 one can prove that this transformation is practically exact for a long-range interaction in a wide region of the coupling strength, including the intermediate and weak-coupling regime and in a wide range of the adiabatic ratio, $\omega / D(\omega$ is the phonon frequency and $D$ is the bandwidth). By applying the Lang-Firsov canonical transformation the hole Matsubara GF is expressed as a convolution of the coherent polaren GF and the multiphonon correlation function $\sigma\left(\mathbf{m}, \Omega_{n}\right) 3^{3}$ 


$$
\mathcal{G}\left(\mathbf{k}, \omega_{n}\right)=\frac{T}{N} \sum_{\omega_{n^{\prime}}, \mathbf{m}, \mathbf{k}^{\prime}} \frac{\sigma\left(\mathbf{m}, \omega_{n^{\prime}}-\omega_{n}\right) e^{i\left(\mathbf{k}-\mathbf{k}^{\prime}\right) \cdot \mathbf{m}}}{i \omega_{n^{\prime}}-\xi_{\mathbf{k}^{\prime}}},
$$

where the multi-phonon correlation function, $\sigma(\mathbf{m}, \tau)=$ $T \sum_{n} e^{-i \Omega_{n} \tau} \sigma\left(\mathbf{m}, \Omega_{n}\right)$ is found as

$$
\sigma(\mathbf{m}, \tau)=\exp \left(\frac{1}{2 N} \sum_{\mathbf{q}}|\gamma(\mathbf{q})|^{2} f_{\mathbf{q}}(\mathbf{m}, \tau)\right) .
$$

Here

$$
\begin{aligned}
f_{\mathbf{q}}(\mathbf{m}, \tau) & =\left[\cos (\mathbf{q} \cdot \mathbf{m}) \cosh \left(\omega_{\mathbf{q}}|\tau|\right)-1\right] \operatorname{coth} \frac{\omega_{\mathbf{q}}}{2 T} \\
& +\cos (\mathbf{q} \cdot \mathbf{m}) \sinh \left(\omega_{\mathbf{q}}|\tau|\right)
\end{aligned}
$$

with $\mathbf{m}$ the lattice vector, $\omega_{n}=\pi T(2 n+1), n=$ $0, \pm 1, \pm 2 \ldots$ and $\Omega_{n}=2 \pi T n$.

In the case of dispersionless phonons and a short-range (Holstein) interaction with a $q$-independent matrix element $\left(\omega_{\mathbf{q}}=\omega,|\gamma(\mathbf{q})|^{2}=2 g^{2}\right)$ one can readjly calculate the Fourier component of $\sigma(\mathbf{m}, \tau)$ to obtain $\mathbf{B}^{3}$

$$
\begin{aligned}
\mathcal{G}\left(\mathbf{k}, \omega_{n}\right) & =\frac{e^{-g^{2}}}{i \omega_{n}-\xi_{\mathbf{k}}}+\frac{e^{-g^{2}}}{N} \sum_{l=1}^{\infty} \frac{g^{2 l}}{l !} \\
& \times \sum_{\mathbf{k}^{\prime}}\left(\frac{n_{\mathbf{k}^{\prime}}}{i \omega_{n}-\xi_{\mathbf{k}^{\prime}}+l \omega}+\frac{1-n_{\mathbf{k}^{\prime}}}{i \omega_{n}-\xi_{\mathbf{k}^{\prime}}-l \omega}\right) .
\end{aligned}
$$

The Green's function of a polaronic carrier comprises two different contributions. The first coherent $\mathbf{k}$-dependent term arises from the polaron band tunneling. The spectral weight of the coherent part is strongly (exponentially) suppressed as $Z=\exp \left(-g^{2}\right)$ and the effective mass is strongly enhanced, $\xi_{\mathbf{k}}=Z E_{\mathbf{k}}-\mu$ (we include the polaronic level shift into the chemical potential, $\mu$ ) - Here $E(\mathbf{k})$ is the bare $(\mathrm{LDA}+\mathrm{U})$ hole band dispersion. The second $\mathbf{k}$-independent contribution describes the excitations accompanied by the emission and absorption of phonons. We believe that this term, $I_{\text {incoh }}(E)$ is responsible for the asymmetric background in the optical conductivity and in the photoemission spectra of cuprates and manganites. We notice that its spectral density spreads over a wide energy range of about twice the polaron level shift $E_{p}=g^{2} \omega$. On the contrary the coherent term shows an angular dependence in the energy range of the order of the polaron bandwidth $2 w \equiv Z D$.

As we have discussed above ARPES measures the imaginary part of the retarded GF multiplied by the Fermi-Dirac distribution function and by the square of the dipole matrix element. $G^{R}$ is obtained from $\mathcal{G}\left(\mathbf{k}, \omega_{n}\right)$ with a substitution $i \omega_{n} \rightarrow E+i 0^{+}$. As a result we obtain

$$
I(\mathbf{k}, E) \sim|d(\mathbf{k})|^{2} n(E) Z \delta\left(E+\xi_{\mathbf{k}}\right)+I_{\text {incoh }}(E),
$$

where $I_{\text {incoh }}(E)$ is a structureless function of the binding energy, which spreads from about $-\omega$ down to $-2 E_{p}$. Only in the extreme limit of a very strong electronphonon coupling, where the polaron bandwidth is well below the phonon frequency, can multiphonon structure in $I_{\text {incoh }}(E)$ be verified. If has actually been observed in the optical conductivity 25 .

The small Holstein polaron is very heavy except if the phonon frequency is extremely high26. In fact, oxides are ionic semiconductors where the long-range electronphonon interaction dominates. That leads to a much lighter mobile small Fröhlich polaron (SFP) 26.38. Considering the electron-phonon interaction in a multi-polaron system one has to take into account the dynamic properties of the dielectric response function 39 . One can naively believe 40 that the long-range Fröhlich interaction becomes short range (Holstein) due to screening. This is of course untrue. In the long-wave limit $(q \rightarrow 0)$ the response of polarons at the optical phonon frequency is dynamic, because $\omega>>q v$ ( $v$ is the characteristic group velocity of polarons). Therefore, the singular (Fröhlich) behaviour of $\gamma(\mathbf{q}) \sim 1 / q$ is unaffected by the screening. Polarons are slow enough and cannot screen the highfrequency crystal field oscillations. Hence, the interaction with the high-frequency optical phonons in ionic polaron solids remains lopo-range whatever the carrier density. It is easy to show 13 using Eq.(5) and Eq.(6) that for any finite-radius interaction with a $q$-dependent matrix element the coherent part of ARPES spectra takes the same form as Eq.(8), but with a different spectral weight $(Z)$ and effective mass $\left(Z^{\prime}\right)$ renormalisation exponents. Also some $\mathbf{k}$ dependence of the incoherent background appears if the matrix element of the electron-phonon interaction depends on $q$ t2. Hence, in general,

$$
I(\mathbf{k}, E) \sim|d(\mathbf{k})|^{2} n(E) Z \delta\left(E+\xi_{\mathbf{k}}\right)+I_{\text {incoh }}(\mathbf{k}, E),
$$

with the same $Z=\exp \left(-E_{p} / \omega\right)$ as in the case of the Holstein polaron but with the SFP bandwidth much less reduced, $\xi_{\mathbf{k}}=Z^{\prime} E(\mathbf{k})-\mu$, where $Z^{\prime}=\exp \left(-\gamma E_{p} / \omega\right)$. In general one finds

$$
\gamma=\sum_{\mathbf{q}}|\gamma(\mathbf{q})|^{2}[1-\cos (\mathbf{q} \cdot \mathbf{a})] / \sum_{\mathbf{q}}|\gamma(\mathbf{q})|^{2},
$$

and $E_{p}=(1 / 2) \sum_{\mathbf{q}}|\gamma(\mathbf{q})|^{2} \omega_{\mathbf{q}}$. The numerical coefficient $\gamma$ might be as small as 0.488 and even smaller in the cuprates with the nearest neighbour oxygen-oxygen distance less than the lattice constant, $\gamma \simeq 0.226$, which is precisely confirmed by exact QMC calculation of the small polaron mass 38 . On the one hand this important result tells us that small polarons as well as intersite bipolarons are perfectly mobile and can account for the high$\mathrm{T}_{c}$ values in cuprates 35 . On the other the coherent spectral weight remains strongly suppressed in polaronic conductors, Eq.(9), because $Z$ might be less than $Z^{\prime}$ by one or even a few orders of magnitude. These unusual SFP spectral features provide an explanation for the apparent discrepancy between a very small Drude weight and a relatively moderate mass enhancement, $m^{*} \sim 3 m_{r}-10 m_{e}$ (depending on doping) of carriers in manganites $\$ 3,44$ and cuprates They also explain why the evHs observed in ARPES20 22 can be hardly seen in angle averaged photoemission. Indeed, the integrated spectral weight of the 
incoherent background is proportional to $(1-Z)$, Eq.(7). It turns out to be much larger than the coherent contribution, proportional to $Z<<1$. Finally, the $\mathbf{k}$ dependent incoherent background might obscure the experimental determination of the Fermi-level crossing, leaving the 'leading edge' determination of it 18 rather unreliable.

In the following we concentrate on the angular, spectral and polarisation dependence of the first coherent term, Eq.(9). The present experimental resolutiont allows probing of the intrinsic damping of the coherent polaron tunnelling. This damping appears due to the random field and low-frequency lattice and spin fluctuations described by the polaron self-energy $\Sigma_{p}(\mathbf{k}, E)$, so that the coherent part of the spectral function is given by

$A_{p}(\mathbf{k}, E)=-(1 / \pi) \frac{\Im \Sigma_{p}(\mathbf{k}, E)}{\left.\left[E+\Re \Sigma_{p}(\mathbf{k}, E)-\xi_{\mathbf{k}}\right)\right]^{2}+\left[\Im \Sigma_{p}(\mathbf{k}, E)\right]^{2}}$.

Hence, the theory of the narrow ARPES peak reduces to the determination of the self-energy of the coherent small hole polaron scattered by impurities, low-frequency deformation and spin fluctuations.

\section{Self-energy of $1 \mathrm{D}$ hole in the non-crossing approximation}

Due to energy conservation small polarons exist in the Bloch states at temperatures well below the optical phonon frequency $T<<\omega / 2$ m matter how strong their interaction with phonons is 3754 47. This textbook result, knewn for a long time, has been questioned by some authors 18 . It has been shown 49 that the confusion is due to a profound misunderstanding of the strong-coupling expansion by those authors. The finite polaron selfenergy appears only due to the (quasi)elastic scattering. First we apply the simplest non-crossing (ladder) approximation to derive the analytical results, Fig.2. Within this approximation the self-energy is $\mathbf{k}$-independent for a short-range scattering potential like the deformation or a screened impurity potential, so that

$$
\Sigma_{p}(E) \sim \sum_{\mathbf{k}} G_{p}^{R}(\mathbf{k}, E),
$$

with $G_{p}^{R}(\mathbf{k}, E)=\left[E-\xi_{\mathbf{k}}-\Sigma_{p}(E)\right]^{-1}$

The oxygen polaron spectrum is parametrised in the tight-binding model as 26

$$
\xi_{\mathbf{k}}^{x, y}=2\left[t \cos \left(k_{x, y} a\right)-t^{\prime} \cos \left(k_{y, x} a\right)+t_{c} \cos \left(k_{z} d\right)\right]-\mu,
$$

If the oxygen hopping integrals in Eq.(13), reduced by the narrowing effect, are positive, the minima of the polaron bands are found at the Brillouin zone boundary in $\mathrm{X}(\pi, 0)$ and $\mathrm{Y}(0, \pi)$. The wave vectors corresponding to the energy minima belong to the stars with two prongs. Their group has only 1D representations. This means that the spectrum is degenerate with respect to the number of prongs of the star. The spectrum Eq.(13) belongs to the star mith two prongs, and, hence it is a two-fold degenerate 36 . The doublet is degenerate if the hole resides on the apical oxygen50. In general, the degeneracy can be removed due to the chains in the buffer layers of Y123 and Y124, so that the $y$-polaronic band corresponding to the tunneling along the chains might be the lowest one.

As mentioned above the oxygen hole is (quasi) onedimensional due to a large difference between the oxygen hopping integrals for the orbitals elongated parallel to and perpendicular to the oxygen-oxygen hopping $t^{\prime}, t_{c}<<t$. This allows us to apply a one-dimensional approximation, reducing Eq.(13) to two 1D parabolic bands near the $X$ and $Y$ points, $\xi_{\mathbf{k}}^{x, y}=k^{2} / 2 m^{*}-\mu$ with $m^{*}=1 / 2 t a^{2}$ and $k$ taking relative to $(\pi, 0)$ and $(0, \pi)$, respectively. Then, the equation for the self-energy in the non-crossing approximation, Eq.(12) takes the following form

$$
\Sigma_{p}(\epsilon)=-2^{-3 / 2}\left[\Sigma_{p}(\epsilon)-\epsilon\right]^{-1 / 2},
$$

for each doublet component. Here we introduce a dimensionless energy (and self-energy), $\epsilon \equiv(E+\mu) / \epsilon_{0}$ using $\epsilon_{0}=\left(D^{2} m^{*}\right)^{1 / 3}$ as the energy unit. The constant $D$ is the second moment of the Gaussian white noise potential, comprising thermal and random fluctuations as $D=2\left(V_{0}^{2} T / M+n_{i m} v^{2}\right)$, where $V_{0}$ is the amplitude of the deformation potential, $M$ is the elastic modulus, $n_{i m}$ is the impurity density, and $v$ is the coefficient of the $\delta$ function impurity potential. The solution is

$$
\begin{aligned}
\Sigma_{p}(\epsilon) & =\frac{\epsilon}{3}-\left(\frac{1+i 3^{1 / 2}}{2}\right)\left[\frac{1}{16}+\frac{\epsilon^{3}}{27}+\left(\frac{1}{256}+\frac{\epsilon^{3}}{216}\right)^{1 / 2}\right]^{1 / 3} \\
& -\left(\frac{1-i 3^{1 / 2}}{2}\right)\left[\frac{1}{16}+\frac{\epsilon^{3}}{27}-\left(\frac{1}{256}+\frac{\epsilon^{3}}{216}\right)^{1 / 2}\right]^{1 / 3}
\end{aligned}
$$

While the energy resplution in the present ARPES studies is almost perfect 4 , the momentum resolution remains rather poor, $\delta>0.1 \pi / a$. Hence we have to integrate the spectral function, Eq.(11), with a Gaussian momentum resolution to obtain the experimental photocurrent,

$$
I(\mathbf{k}, E) \sim Z \int_{-\infty}^{\infty} d k^{\prime} A_{p}\left(k^{\prime},-E\right) \exp \left[-\frac{\left(k-k^{\prime}\right)^{2}}{\delta^{2}}\right] .
$$

The integral is expressed in terms of $\Sigma_{p}(\epsilon)$, Eq.(15) and the tabulated Error function $w(z)$ as

$$
I(\mathbf{k}, E) \sim-\frac{2 Z}{\delta} \Im\left(\Sigma_{p}(\epsilon)\left[w\left(z_{1}\right)+w\left(z_{2}\right)\right]\right),
$$

where $z_{1,2}=\left[ \pm k-i / 2 \Sigma_{p}(\epsilon)\right] / \delta, w(z)=e^{-z^{2}} \operatorname{erfc}(-i z)$ and $\epsilon=(-E+\mu) / \epsilon_{0}$. This photocurrent is plotted as dashed lines in Fig.3 for two momenta, $k=0.04 \pi / a$ (almost $\mathrm{Y}$ or X points of the Brillouin zone) and $k=0.3 \pi / a$. 
The chemical potential is placed in the charge transfer gap below the bottom of the hole band, $\mu=-20 \mathrm{meV}$, the momentum resolution is taken as $\delta=0.28 \pi / a$ and the damping $\epsilon_{0}=19 \mathrm{meV}$.

The imaginary part of the self-energy, Eq.(15) disappears below $\epsilon=-3 / 2^{5 / 3} \simeq-0.9449$. Hence this approximation gives a well-defined gap rather than a pseudogap. Actually, the non-crossing approximation fails to describe the localised states inside the gap (i.e. the Lifshitz tail of the density of states). One has to go beyond the simple ladder, Fig.2, the describe the single-electron tunnelling inside the gap 32 and the ARPES spectra at very small binding energy.

\section{Exact spectral function of 1D hole}

The exact spectral function for a one dimensional particle in a random Gaussian white noise potential was derived by Halperin27 and the density of states by Frisch and Lloyd51. Halperin derived two pairs of differential equations from whose solutions the spectral function of a 'Schrodinger' particle (i.e. in the effective mass approximation) and of a 'discrete' particle (tight-binding approximation) may be calculated. The QMC polaronic bandwidth is about $100 \mathrm{meV}$ or larger 38 , which allows us to apply the 'Schrodinger' particle spectral function, given by 27

$$
A_{p}(k, \epsilon)=4 \int_{-\infty}^{\infty} p_{0}(-z) \Re p_{1}(z) d z,
$$

where $p_{0,1}(z)$ obey the two differential equations

$$
\left[\frac{d^{2}}{d z^{2}}+\frac{d}{d z}\left(z^{2}+2 \epsilon\right)\right] p_{0}=0
$$

and

$$
\left[\frac{d^{2}}{d z^{2}}+\frac{d}{d z}\left(z^{2}+2 \epsilon\right)-z-i k\right] p_{1}+p_{0}=0
$$

with boundary conditions

$$
\lim _{z \rightarrow \infty} z^{2-n} p_{n}(z)=\lim _{z \rightarrow-\infty} z^{2-n} p_{n}(z)
$$

where $k$ is measured in units of $k_{0}=\left(D^{1 / 2} m^{*}\right)^{2 / 3}$. The first equation may be integrated to give

$p_{0}(z)=\frac{\exp \left(-z^{3} / 3-2 z \epsilon\right) \int_{-\infty}^{z} \exp \left(u^{3} / 3+2 u \epsilon\right) d u}{\pi^{1 / 2} \int_{0}^{\infty} u^{-1 / 2} \exp \left(-u^{3} / 12-2 u \epsilon\right) d u}$.

The equation for $p_{1}(z)$ has no known analytic solution, and hence must be solved numerically. There is however an asymptotic expression for $A_{p}(k, \epsilon)$ in the tail where $|\epsilon| \gg 1$ :

$$
A_{p}(k, \epsilon) \sim 2 \pi(-2 \epsilon)^{\frac{1}{2}} \exp \left[-\frac{4}{3}(-2 \epsilon)^{\frac{3}{2}}\right] \cosh ^{2}\left[\frac{\pi k}{(-8 \epsilon)^{\frac{1}{2}}}\right] .
$$

In practice, for computational efficiency we use the exact spectral density for $-1.4 \leq \epsilon<1$, and outside this range we use the asymptotic result for $\epsilon<-1.4$, Eq.(23) and the non-crossing approximation for $\epsilon \geq 1$, where they are almost indistinguishable from the exact result on the scale of the diagrams plotted here.

The result for $A_{p}(k,-E)$ integrated with the Gaussian momentum resolution is shown in Fig.3 for two values of the momentum (solid lines). Quite differently from the non-crossing approximation the exact spectral function (averaged with the momentum resolution function) has the Lifshitz tail due to the states localised by disorder within the normal state gap. However, besides this tail the non-crossing approximation gives very good agreement, and for binding energy greater than about $30 \mathrm{meV}$ it is practically exact.

The cumulative DOS

$$
N_{p}(\epsilon)=(2 \pi)^{-1} \int_{-\infty}^{\epsilon} d \epsilon^{\prime} \int_{-\infty}^{\infty} d k A_{p}\left(k, \epsilon^{\prime}\right)
$$

is expressed analytically 51 in terms of the tabulated Airy functions $A i(x)$ and $B i(x)$ as

$$
\left.N_{p}(\epsilon)\right)=\pi^{-2}\left[A i^{2}(-2 \epsilon)+B i^{2}(-2 \epsilon)\right]^{-1} .
$$

The DOS $d N(\epsilon) / d \epsilon$ fits well the voltage-current tunnelling characteristics of cuprate 32 .

\section{Theory of ARPES in Y124 and Y123}

With the polaronic doublet, Eq.(13) placed above the chemical potential we can mlantitatively describe highresolution ARPES in Y12321 and Y12420. First we explain the experimentally observed polarisation dependence of the intensity near $\mathrm{Y}$ and $\mathrm{X} 21$. The Bloch periodic function $u_{\mathbf{k}}(\mathbf{r})$ can be expressed in terms of the Wannier orbitals $w(\mathbf{r})$ as

$$
u_{\mathbf{k}}(\mathbf{r})=N^{-1 / 2} \sum_{\mathbf{m}} e^{i \mathbf{k} \cdot(\mathbf{m}-\mathbf{r})} w(\mathbf{r}-\mathbf{m}) .
$$

Then the dipole matrix element is given by the derivative of the Fourier component of the atomic (Wannier) orbital, $f_{\mathbf{k}} \equiv v_{0}^{-1 / 2} \int d \mathbf{r} w(\mathbf{r}) \exp (i \mathbf{k} \cdot \mathbf{r})$ as

$$
\mathbf{d}(\mathbf{k})=i\left(\mathbf{e} \cdot \nabla_{\mathbf{k}}\right) f_{\mathbf{k}}
$$

To estimate $f_{\mathbf{k}}$ we approximate the $x, y$ oxygen orbitals contributing to the $\mathrm{x}$ and $\mathrm{y}$ polaronic bands, respectively, with $w_{x}(\mathbf{r})=\left(1 / 8 a_{0}^{3} \pi\right)^{1 / 2}\left(x / 2 a_{0}\right) \exp \left(-r / 2 a_{0}\right)$ and $w_{y}(\mathbf{r})=\left(1 / 8 a_{0}^{3} \pi\right)^{1 / 2}\left(y / 2 a_{0}\right) \exp \left(-r / 2 a_{0}\right)$. As a result we obtain for the $\mathrm{x}$ orbital,

$$
\begin{aligned}
\frac{\partial f_{\mathbf{k}}}{\partial k_{x}} & =\left(8 a_{0}^{3} \pi / v_{0}\right)^{1 / 2} a_{0} \\
& \times\left(\left[\left(k a_{0}\right)^{2}+1 / 4\right]^{-3}-6\left(k_{x} a_{0}\right)^{2}\left[\left(k a_{0}\right)^{2}+1 / 4\right]^{-4}\right),
\end{aligned}
$$

and

$$
\frac{\partial f_{\mathbf{k}}}{\partial k_{y, z}}=-6\left(8 a_{0}^{3} \pi / v_{0}\right)^{1 / 2} a_{0}^{3} k_{x} k_{y, z}\left[\left(k a_{0}\right)^{2}+1 / 4\right]^{-4},
$$


Here $\mathbf{k}$ is the photoelectron momentum and $a_{0}$ is the size of the Wannier function. For the case of y-orbital one should interchange $\mathrm{x}$ and $\mathrm{y}$. Near the $\mathrm{X}$ and $\mathrm{Y}$ points of the Brillouin zone, $\left|k_{y, x}\right|<<k$, respectively. Then it follows from Eq.(28) and Eq.(29) that the ARPES peak should be seen at $\mathrm{X}$ and almost disappear at $\mathrm{Y}$ if the photons are polarised along the $\mathrm{x}$-direction, i.e. e $\| \mathbf{a}$. If the polarisation is along the $y$-direction $(\mathbf{e} \| \mathbf{b})$ the peak appears at $\mathrm{Y}$ and almost disappears at X. Precisely this behaviour is observed in ARPES spectra obtained using polarised photons 21 , Fig.4. We also notice a very strong dependence of the dipole matrix element, Eq.(28) on the photon energy, $d \sim \nu^{-3}$ at large $\nu$. Hence, it is not surprising if the ARPES pak disappears at large $\nu$ as has been recently observed 52 .

The exact 1D polaron spectral function, Eq.(18), integrated with the experimental momentum resolution, provides a quantitative fit to the ARPES spectra in Y124 along the $Y-\Gamma$ direction, as shown in Fig.5. The angular dispersion is described with the polaron mass $m^{*}=9.9 m_{e}$ in agreement with the Monte-Carlo calculations of the SFP mass 8 . The spectral shape is reproduced well with $\epsilon_{0}=19 \mathrm{meV}$, Fig.6, in close agreement with the value of this parameter found in tunnelling experiments32. That yields an estimate of the polaron scattering rate, which appears to be smaller than the polaron bandwidth (about $100 \mathrm{meV}$ or larger), in agreement with the notion 26 that many high- $\mathrm{T}_{c}$ cuprates are in the clean limit. There is also quantitative agreement in the perpendicular direction $Y-S$, Fig.7, in a restricted region of small $k_{x}$, where almost no dispersion is observed around Y. Slight dispersion in the $Y-S$ direction towards the chemical potential might be due to a negative $t^{\prime}$ in Eq.(13).

However, there is a significant loss of the energyintegrated intensity along both directions, Fig. 8, which the theoretical spectral function alone cannot account for. The energy-integrated ARPES spectra obey the sum rule,

$$
\int_{-\infty}^{\infty} d E I(\mathbf{k}, E) \sim|d(\mathbf{k})|^{2} n_{\mathbf{k}}
$$

where $n_{\mathbf{k}}=\left\langle h_{\mathbf{k}} h_{\mathbf{k}}^{\dagger}\right\rangle$. If the dipole matrix element is almost $\mathrm{k}$-independent and the chemical potential is pinned well inside the charge-transfer gap, so that $n_{\mathbf{k}}=1$ this integral would be $\mathbf{k}$ independent as well. This is not the case for Y124, no matter what the scanning direction is, Fig.8. Therefore, we have to conclude that either the dipole matrix element is $\mathbf{k}$ dependent or (and) the oxygen band is strongly correlated (in the Mott-Hubbard sense). As we have mentioned above, the incoherent background of SFP is angle dependent as well, which might contribute to the intensity loss.

The rapid loss of the integrated intensity in the $Y-S$ direction was interpreted by Randeria and Campuzano 18 as a Fermi-surface crossing. While a Fermi-surface crossing is not incompatible with our scenario (see Fig.1 in- set), we do not believe that it has really been observed in Y124. First of all these authors suppressed a few experimental curves in the $Y-\Gamma$ direction, Fig.5, which prevented them from observing the intensity loss in this 'dielectric' direction, where there is obviously no Fermisurface crossing. This loss of intensity along $Y-\Gamma$ tells us that the intensity loss might be due to the matrix element rather than to the Fermi-surface crossing in both directions. This is confirmed by our observation of a similar rapid loss of the intensity in a dielectric, FeSi23, Fig.9 with no Fermi-surface at all. The peaks in the $Y-S$ direction are all $15 \mathrm{meV}$ or more below the Fermi level - at a temperature of $1 \mathrm{meV}$, if the loss of spectral weight were due to a Fermi-surface crossing one would expect the peaks to approach much closer to the Fermi level. Also the experimental spectral shape of the intensity at $\mathbf{k}=\mathbf{k}_{F}$ is incompatible with any theoretical scenario, including different marginal Fermi-liquid models, Fig.10. The spectral function on the Fermi surface should be close to a simple Lorentzian,

$$
A_{p}\left(\mathbf{k}_{F}, E\right) \sim \frac{|E|^{\beta}}{E^{2}+\text { constant } \times E^{2 \beta}},
$$

because the imaginary part of the self-energy behaves as $|E|^{\beta}$ with $0 \leq \beta \leq 2$. On the contrary, the experimental intensity shows a pronounced minimum at the alleged Fermi-surface, Fig.10.

If there is indeed no Fermi-surface crossing in many cuprates, as we argue, why then does the "maximum locus' determination point to a large Fermi surface in cuprates, which is drastically incompatible with their kinetic and thermodynamic properties? We propose that it appears due to the fact that oxygen semiconducting band has its minima at large $k$ inside or even on the boundary of the Brillouin zone. That is why ARPES show intense peaks near large $\mathbf{k}$ imitating a large Fermi-surface.

\section{Summary and conclusions}

In summary, we have proposed a theory of ARPES in cuprates based on the $\mathrm{LDA}+\mathrm{U}$ band structure and the bipolaron theory compatible with the normal state kinetic and thermodynamic properties of these materials. The theory explains the narrow flat bands observed in Y123 and Y124, including their polarisation, spectral and angular dependence, as well as a featureless (but dispersive) background. The ARPES peak originates from the hole excitations of the polaronic oxygen band of the buffer layers, in agreement with the experimental results and electronic structure of Schabel et al 21 . Differently from these authors we suggest that this band is intrinsic for cuprates and takes part in the bipolaron formation and superconductivity, which is nicely confirmed by a few independent studie 50. The normal state gap is half of the bipolaron binding energy. The angular dependence of the peak and of the gap is due to the polaron band dispersion, which agrees well with the QMC results for the small Fröhlich polaron.

The spectral shape of the peak is affected by the soft lattice, spin and random fluctuations. The characteristic 
scattering rate agrees well with that found in the tunnelling experiments 32 . This scattering rate is temperature dependent not only due to the thermal lattice fluctuations, but also because of the anomalous screening below $\mathrm{T}_{c}$ in the charged Bose-liquida. The Bose-Einstein condensate screens effectively the long-range Coulomb potential of impurities. As a result one can expect a drastic change of the damping $\epsilon_{0}$ when $\mathrm{T}_{c}$ is passed. That might help to understand the near disappearance of the narrow peak above $\mathrm{T}_{c}$ in some Bi-cuprates. On the other hand in the stehiometric Y124 with (theoretically) no impurities, one can expect about the same results from ABPES below and above $\mathrm{T}_{c}$, which seems to be the case 20 . Within the bipolaron theory there is only one single-particle gap, which is half of the bipolaron binding energy both below and above $\mathrm{T}_{c}$.

We believe that many cuprates are doped insulators with no Fermi surface at all due to the bipolaron formation. The Fermi-surface crossing, if it were firmly established in the overdoped samples, would correspond to a small Fermi surface of the oxygen band pockets located at finite $\mathbf{k}$ like in many ordinary semiconductors, for example, in Ge and Si.

The authors greatly appreciate enlightening discussions with D.S. Dessau, N.E. Hussey, V.V. Kabanov, P.E. Kornilovitch, G.J. Kaye, A.I. Lichtenstein, G.A. Sawatzky, J.R. Schrieffer, Z.-X. Shen, J. Zaanen, G. Zhao, and R. Zeyher. C.J.D. has been supported in this work by a grant from the EPSRC of the UK.

${ }^{1}$ J.G. Bednorz and K.A. Müller, Z.Phys. B64, 189 (1986); Angew.Chem.Int.Ed.Engl. 27, 735 (1988).

${ }^{2}$ A.S. Alexandrov and N.F. Mott, Rep. Prog. Phys. 57 1197; 'High Temperature Superconductors and Other Superfluids' Taylor and Francis, London (1994); 'Polarons and Bipolarons', World Scientific, Singapore (1995).

${ }^{3}$ A.S. Alexandrov, in 'Models and Phenomenology for Conventional and High-temperature Superconductivity' (Course CXXXVI of the Intenational School of Physics 'Enrico Fermi'), eds. G. Iadonisi, J.R. Schrieffer and M.L. Chiofalo, IOS Press (Amsterdam), p. 309 (1998).

${ }^{4}$ for a recent review see Zhi-xun Shen, in 'Models and Phenomenology for Conventional and High-temperature Superconductivity' (Course CXXXVI of the Intenational School of Physics 'Enrico Fermi'), eds. G. Iadonisi, J.R. Schrieffer and M.L. Chiofalo, IOS Press (Amsterdam), p. 141 (1998).

${ }^{5}$ D.C. Johnston, Phys. Rev. Lett 62, 957 (1989).

${ }^{6}$ K.A. Müller et al, J.Phys.: Condens. Matter 10, L291 (1998).

${ }^{7}$ D. Mihailovi'c et al, J. Superconductivity, 10, 337 (1997).

8 J. Hofer et al, Physica C 297, 103 (1998).

${ }^{9}$ B. Batlogg et al, Physica C 135 - 140, 130 (1994); H.Y. Hwang et al, Phys. Rev. Lett. 72, 2636 (1994)
10 J.W. Loram et al, Physica C (Amsterdam), 235, 134 (1994).

${ }^{11}$ C. Kendziora et al, Phys. Rev. Lett. 79, 4935 (1997).

12 Z.-X. Shen and J.R. Schrieffer, Phys. Rev. Lett. 78, 1771 (1997) and references therein.

${ }^{13}$ Ch. Renner et al, Phys. Rev. Lett. 80, 149 (1998).

14 N.L. Saini et al, Phys. Rev. Lett. 79, 3467 (1997).

15 N.L. Saini et al, Phys. Rev. Lett. 82, 2619 (1999)

16 J.W. Loram et al, J. of Superconductivity 7, 243 (1994).

${ }^{17}$ H. Ding et al, Nature (London) 382, 51 (1996).

${ }^{18}$ M. Randeria and J.-C. Campuzano, in 'Models and Phenomenology for Conventional and High-temperature Superconductivity' (Course CXXXVI of the Intenational School of Physics 'Enrico Fermi'), eds. G. Iadonisi, J.R. Schrieffer and M.L. Chiofalo, IOS Press (Amsterdam), p. 115 (1998).

19 J. Mesot et al, Phys. Rev. Lett. 82, 2619 (1999).

${ }^{20}$ K. Gofron et al, Phys. Rev. Lett. 73, 3302 (1994).

${ }^{21}$ M. C. Schabel et al, Phys. Rev. B 57, 6090 (1998).

22 A. Ino et al, unpublished.

${ }^{23}$ C.-H. Park et al, Phys. Rev. B 52, R16981 (1995).

24 A.S. Alexandrov, Philos. Trans. R. Soc. London, Ser. A356, 197 (1998).

${ }^{25}$ see in 'Anharmonic Properties of High- $T_{c}$ Cuprates', eds. D. Mihailovi'c, G. Ruani, E. Kaldis and K.A. Müller, World Scientific, Singapore (1995), and also in 'Polarons and Bipolarons in High- $T_{c}$ Superconductors and Related Materials', eds. E.K.H. Salje, A.S. Alexandrov and W.Y. Liang , Cambridge University Press, Cambridge (1995) .

${ }^{26}$ A.S. Alexandrov, Phys. Rev. B53, 2863 (1996).

${ }^{27}$ B.I. Halperin, Phys. Rev. 139, A104 (1965).

${ }^{28}$ C.R.A. Catlow, M.S. Islam and X. Zhang, J. Phys.: Condens. Matter 10, L49 (1998).

29 A.S. Alexandrov, Physica C (Amsterdam) 182, 327 (1991).

30 A.S. Alexandrov, V.V. Kabanov and N.F. Mott, Phys. Rev. Lett. 77, 4796 (1996).

31 A.S. Alexandrov and G.J Kaye, J. Phys.: Condens. Mattter 11, L15 (1999).

32 A.S. Alexandrov, Physica C (Amsterdam) 305, 46 (1998).

33 G.A. Sawatzky, invited talk at the Rinberg Workshop (Rinberg Castle, Germany), April 1999.

${ }^{34}$ D.A. Wollman et al, Phys. Rev. Lett. 71, 2134 (1993); C.C. Tsuei et al, Phys. Rev. Lett. 73, 593 (1994); J.R. Kirtley et al, Nature 373, 225 (1995); C.C. Tsuei et al, Science 272, 329 (1996).

35 A.S. Alexandrov, Phys. Rev. Lett. 82, 2620 (1999).

36 A.S. Alexandrov and V.V. Kabanov, Phys. Rev. B 59 (1999).

${ }^{37}$ I.G. Lang and Yu.A. Firsov, Zh.Eksp.Teor.Fiz. 43, 1843 (1962) ( Sov.Phys.JETP 16, 1301 (1963)).

38 A.S. Alexandrov and P.E. Kornilovich, Phys. Rev. Lett. 82, 807 (1999).

39 A.S. Alexandrov, Phys. Rev. B 46, 2838 (1992).

${ }^{40}$ B.K. Chakraverty, J. Ranninger, and D. Feinberg. Phys. Rev. Lett. 82, 2621 (1999).

${ }^{41}$ A.S. Alexandrov and C. Sricheewin, unpublished.

${ }^{42}$ G.J. Kaye, Phys. Rev. B 57, 8759 (1998).

${ }^{43}$ Y. Okimoto et al., Phys. Rev. Lett. 75, 109 (1995); Y. Okimoto et al., Phys. Rev. B 55, 4206 (1997).

${ }^{44}$ D.S. Dessau et al, Phys. Rev. Lett. 81, 192 (1998). 
45 T. Holstein, Ann.Phys. 8, 325-42; ibid p. 343 (1959).

46 J. Appel, in Solid State Physics, eds. F. Seitz, D. Turnbull and H. Ehrenreich, Academic Press 21 (1968).

${ }^{47}$ Yu.A. Firsov (ed), Polarons, Nauka (Moscow) (1975).

48 E.V.L. de Mello and J. Ranninger, Phys. Rev. B55, 14872 (1997); ibid B58, 9098 (1998).

${ }^{49}$ Yu.A. Firsov et al, Phys. Rev. B 59, (1999).

50 There is strong experimental evidence for apical holes in cuprates from the site-specific $X$-ray absorption (M. Merz et al, Phys. Rev. Lett. 80, 5192 (1998)) and from the site-specific effect of $B a$ and $Y$ substitution for $\mathrm{Pr}$ in $\mathrm{YBa}_{2} \mathrm{Cu}_{3} \mathrm{O}_{7}$ (J.D. Dow, U. Howard and A. Blackstead, Bulletin of American Physical Society, 43, 877 (1998)).

${ }^{51}$ H.L. Frisch and S.P. Lloyd, Phys. Rev. 120, 1175 (1960).

52 D.S. Dessau et al, cond-mat/9904050.

\section{Figure Captures}

Fig.1. Schematic LDU+U density of states. The chemical potential is pinned inside the charge transfer gap (a) due to the bipolaron formation in underdoped cuprates. It might enter the oxygen band in overdoped cuprates (b) if the polaron band crosses the bipolaron one (inset).

Fig.2. The non-crossing diagram for the self-energy. The dashed line corresponds to the random potential and (or) to the thermal lattice and spin fluctuations.

Fig.3. The polaron spectral function, integrated with the momentum resolution function for two angles, $k=$ $0.04 \pi / a$ (upper curves), and $k=0.30 \pi / a$ with the damping $\epsilon_{0}=19 \mathrm{meV}$, the momentum resolution $\delta=0.28 \pi / a$ and the polaron mass $m^{*}=9.9 m_{e}$. The bipolaron binding energy $2|\mu|=40 \mathrm{meV}$. The dashed curves are the spectral density integrated with the momentum resoltion in the non-crossing approximation.

Fig.4. Polarisation dependence of the ARPES peak in Y12321 near X and $\mathrm{Y}$ points.

Fig.5. Theoretical ARPES spectra (b) compared with experiment (a) in Y12420 for $Y-\Gamma$ direction. Parameters are those of Fig.3.

Fig.6. Theoretical fit (dashed lines) to two experimental ARPES curves corresponding to $k=0.04 \pi / a$ (upper curves), and $k=0.30 \pi / a$.

Fig.7. Theoretical ARPES spectra (b) compared with experiment (a) in Y12420 for $Y-S$ direction.

Fig.8. The energy-integrated ARPES intensity in Y124 in the $Y-\Gamma$ (a) and $Y-S$ (b) directions. Momenta are measured relative to the $\mathrm{Y}$-point of the Brillouin zone.

Fio 9. (a) The energy-integrated ARPES intensity in FeSi23 as a function of the analyser angle. The spectra are shown in (b).

Fig.10. The experimental ARPES signal (solid line) on the alleged Fermi-surface does not correspond to a Fermi-liquid spectral function (dashed line). We assume particle-hole symmetry to obtain the spectral function for negative binding energy. 


\section{LDA+U DOS}

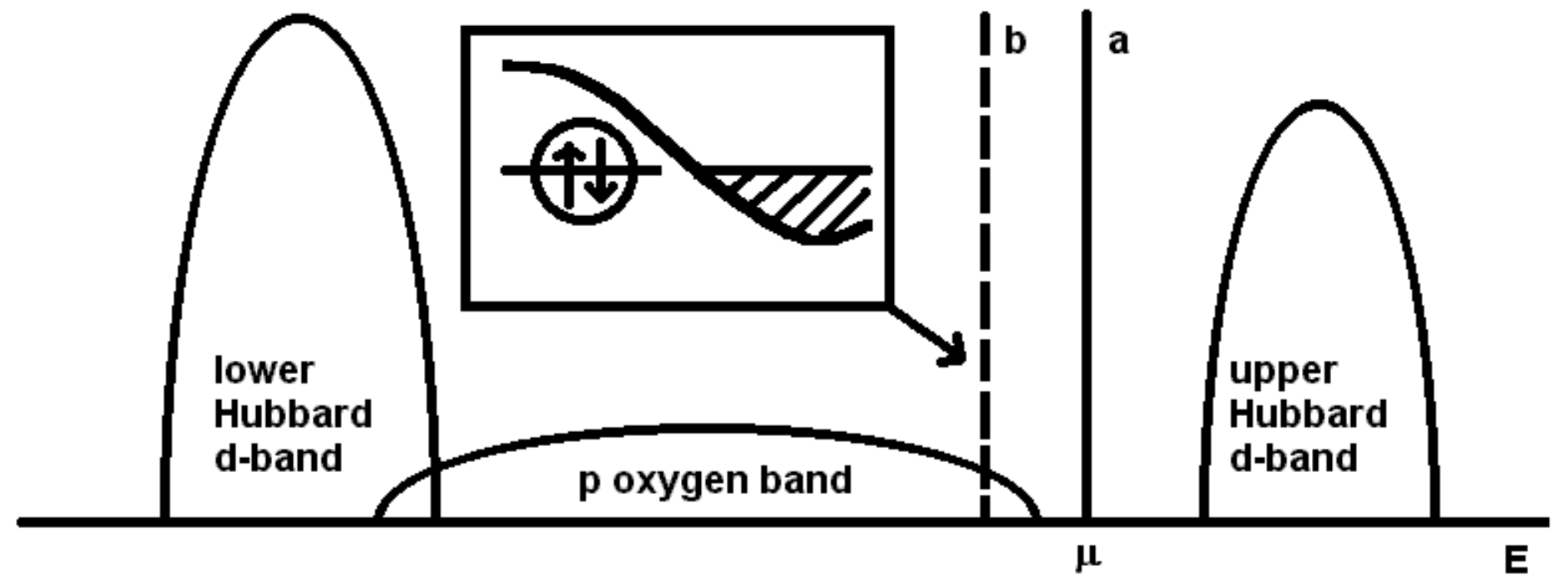


$\frac{M}{I}$

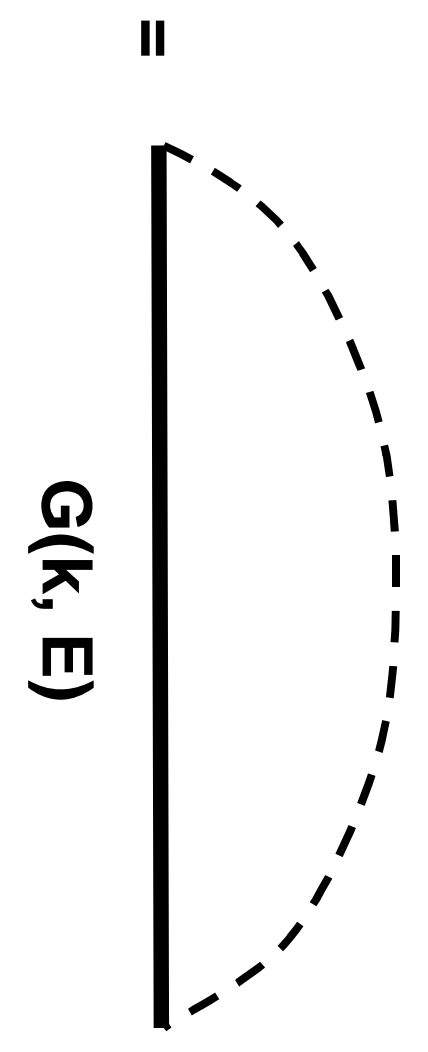




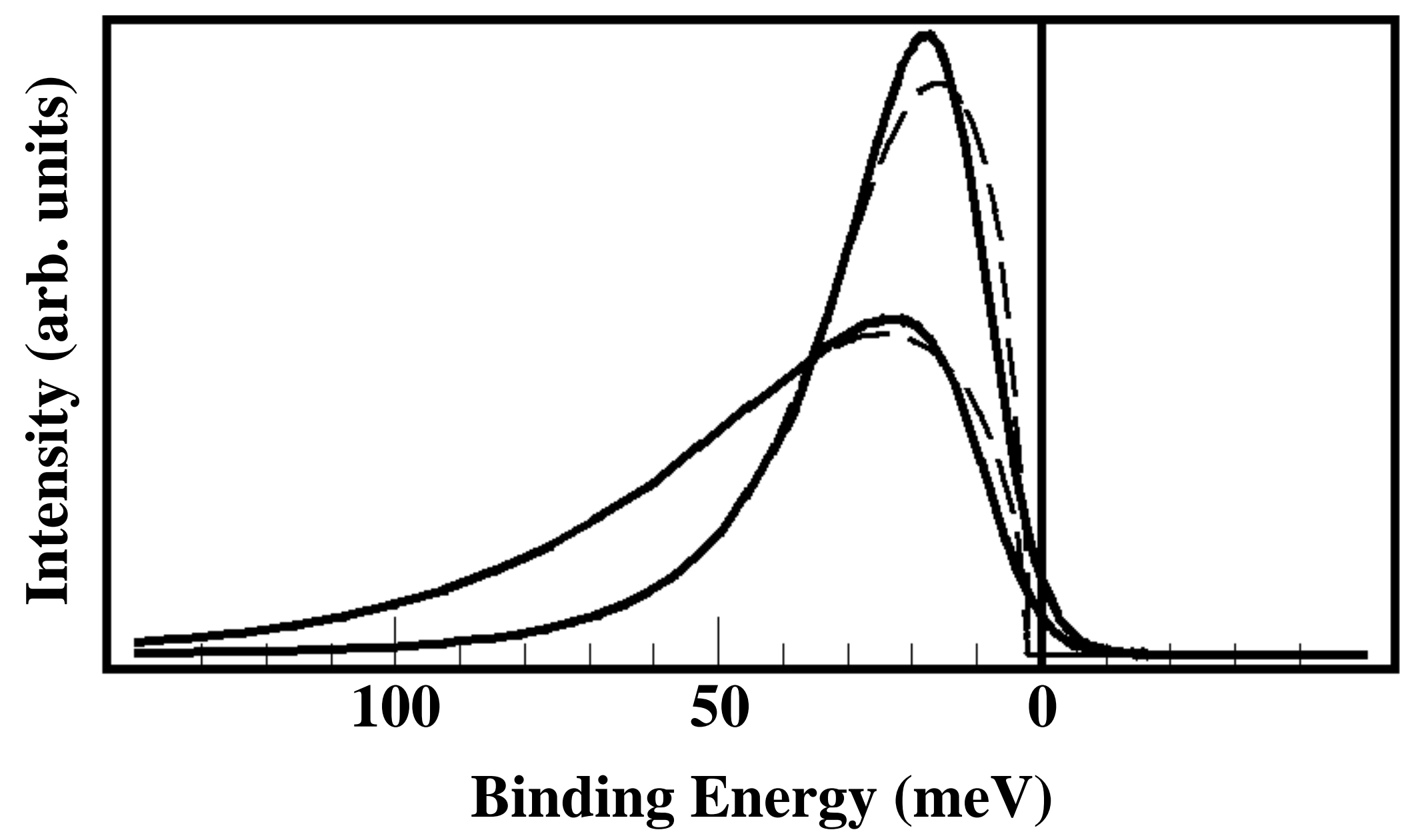


Theory

Experiment

I

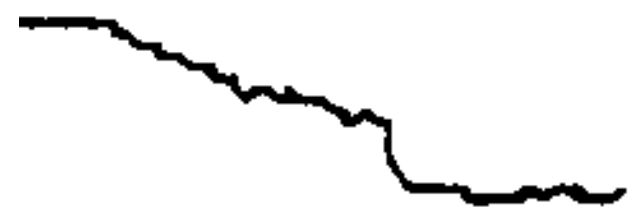

X

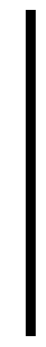

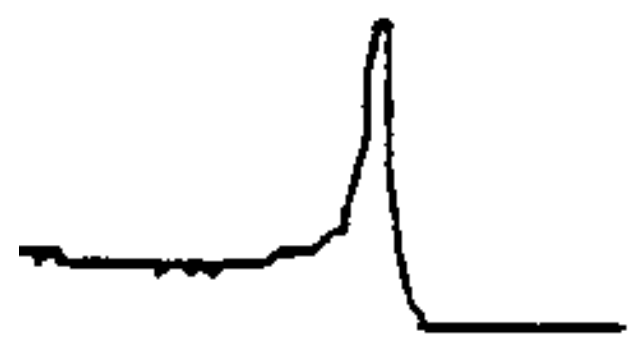

E || b

Y

Binding Energy
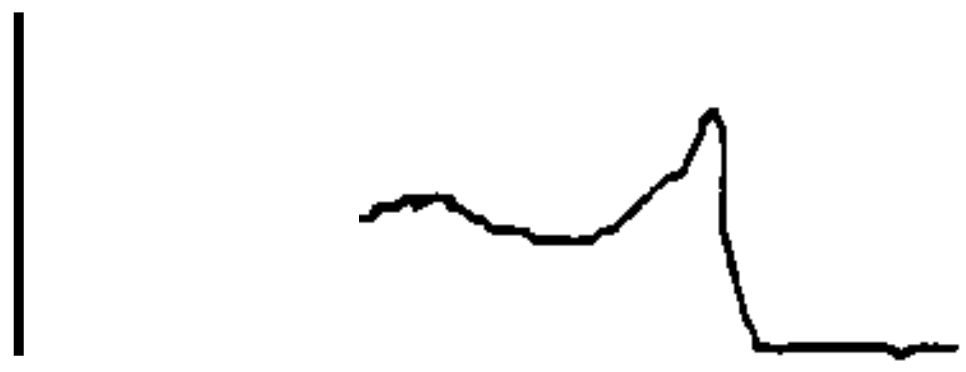

$\mathbf{E} \| \mathbf{a}$

I
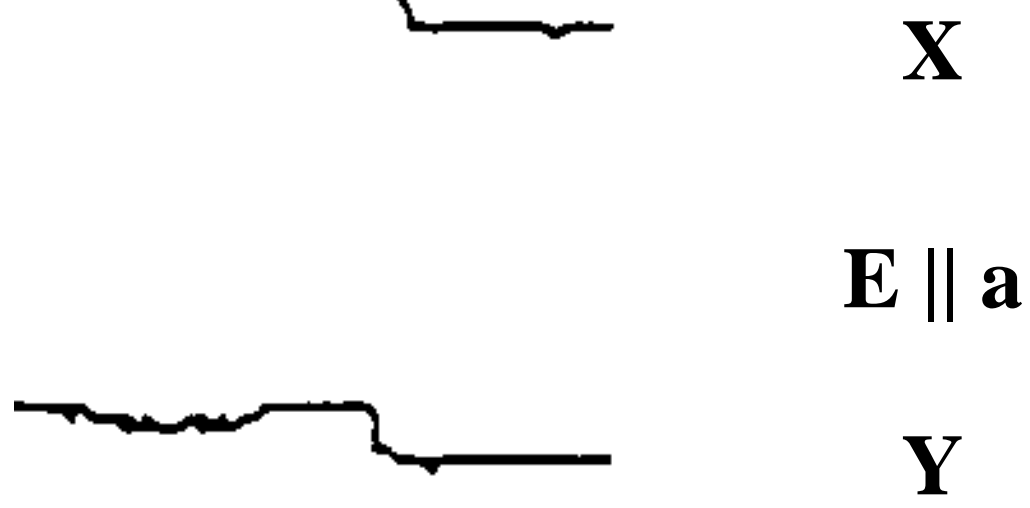

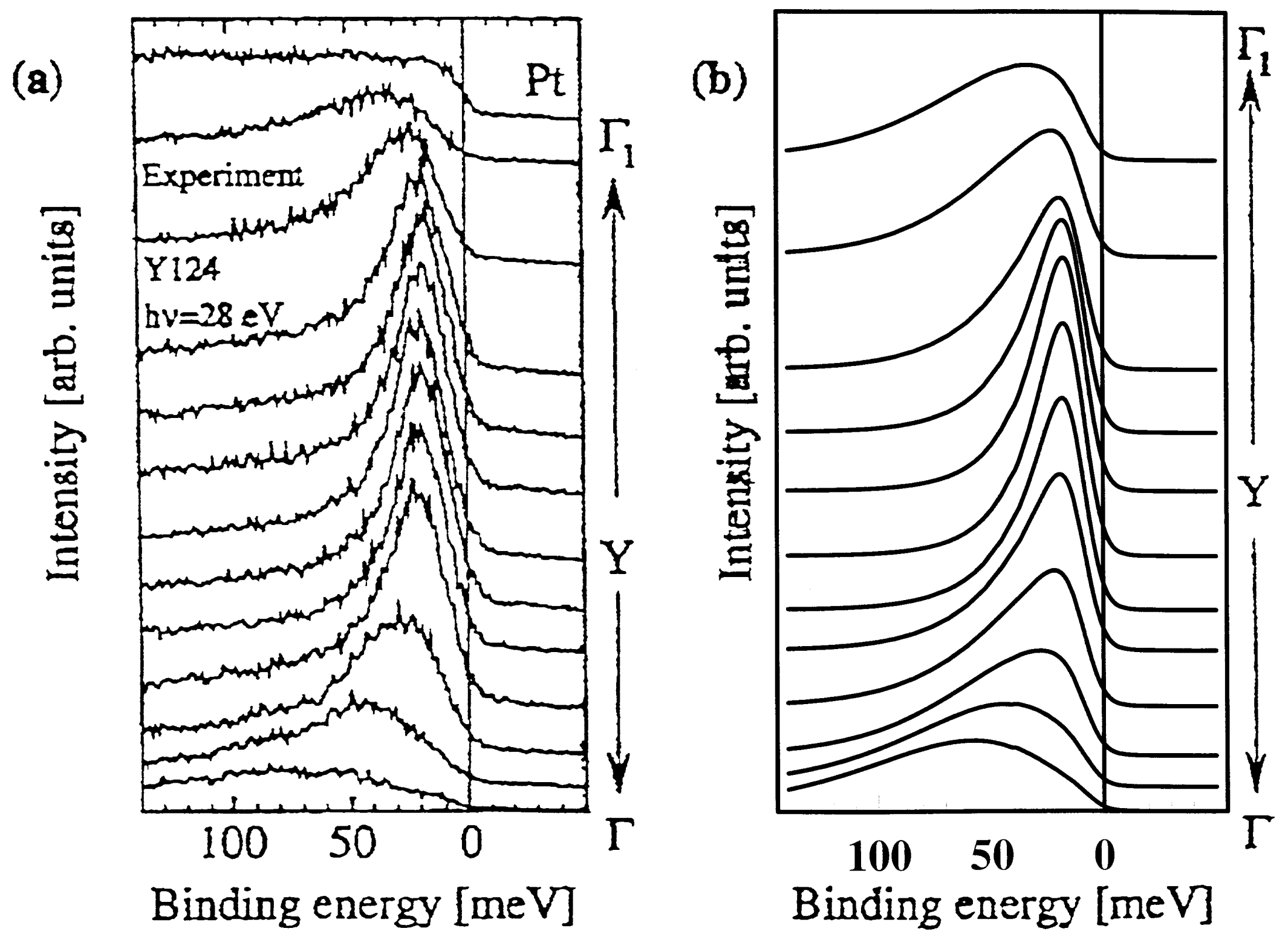
Intensity (arb. units)

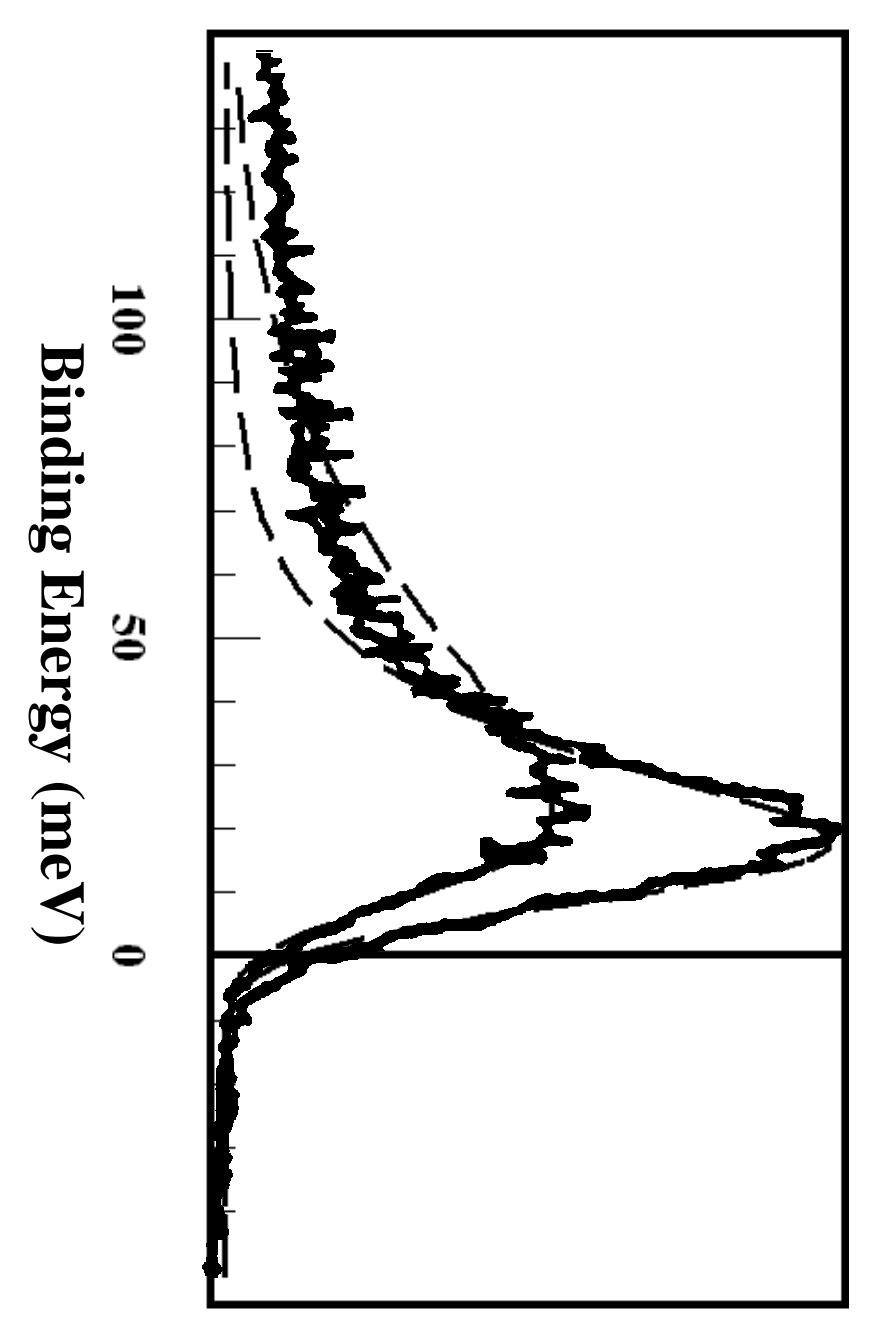




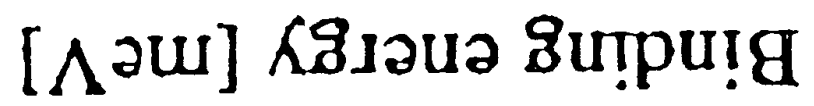

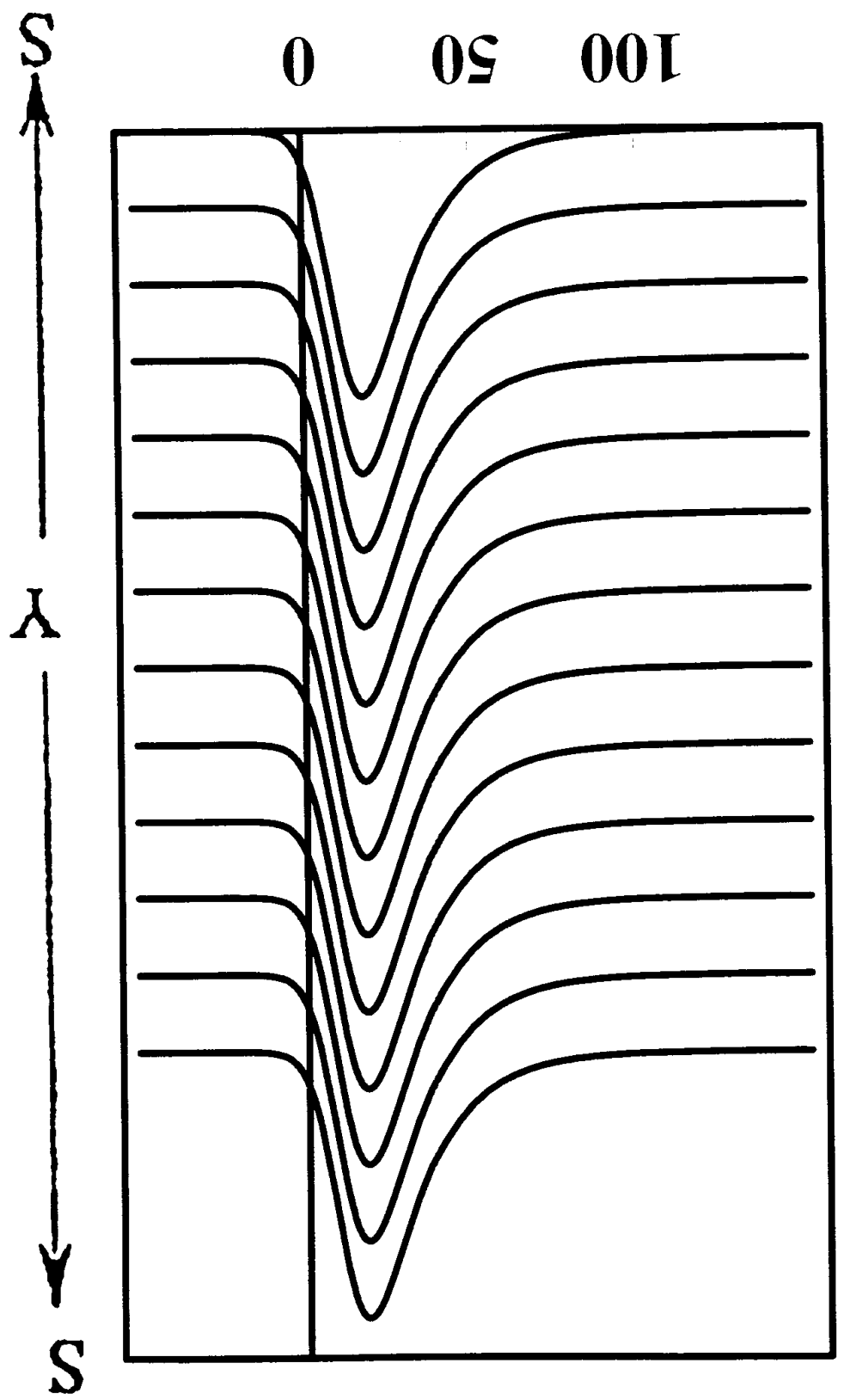

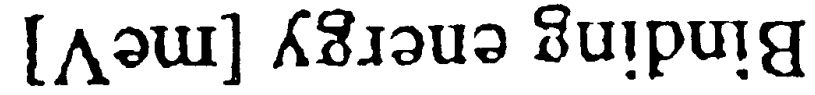

$S \quad 0$ OS 00I

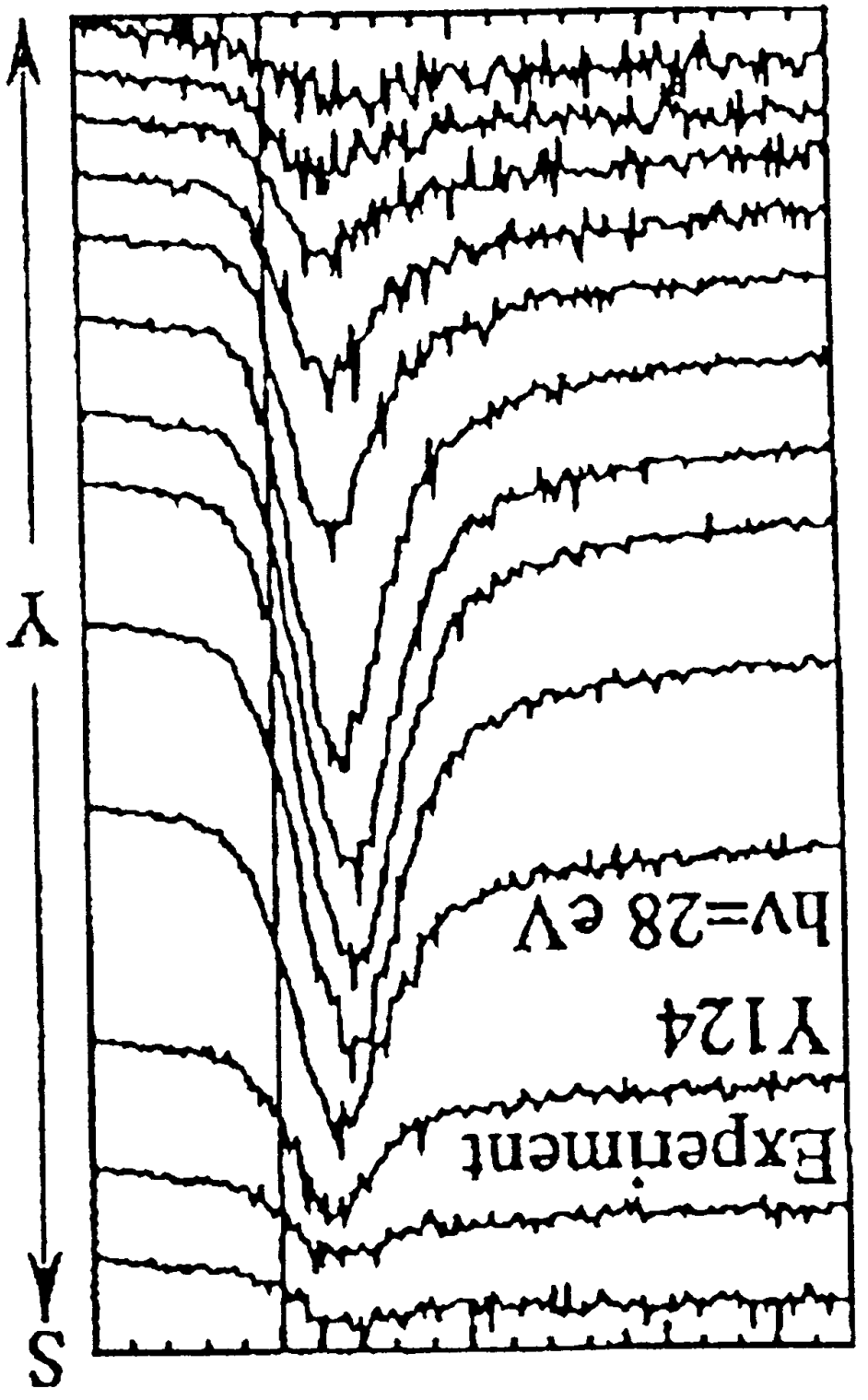

(q)

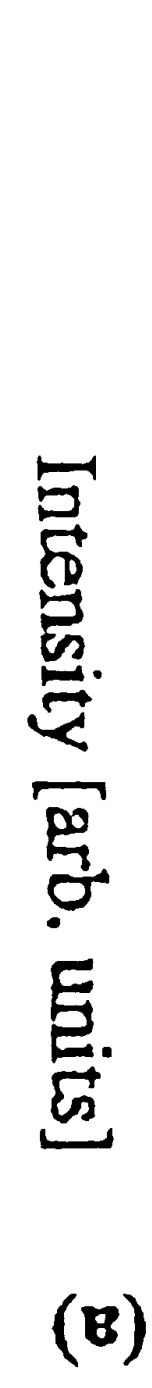


(a)

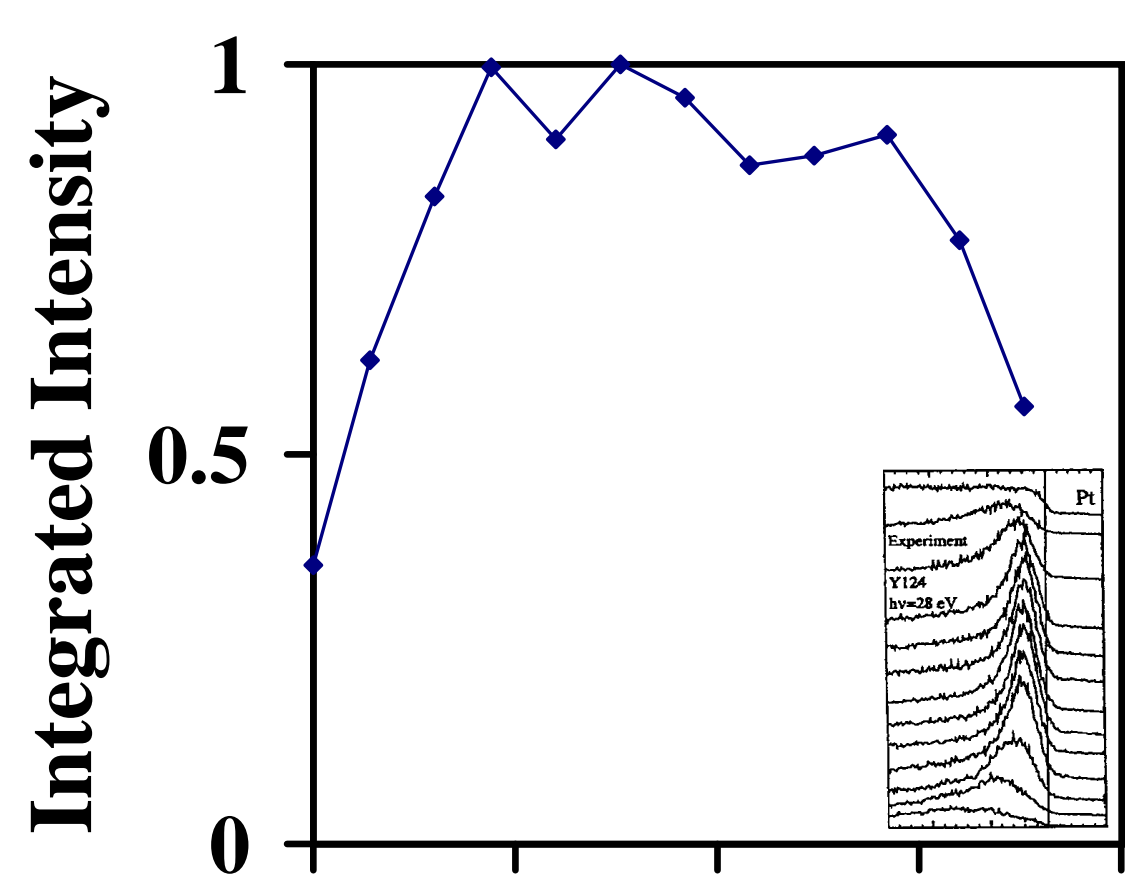

$\begin{array}{lllll}-0.5 & -0.25 & 0 & 0.25 & 0.5\end{array}$ $\mathrm{ky}(\pi / \mathrm{a})$ (b)

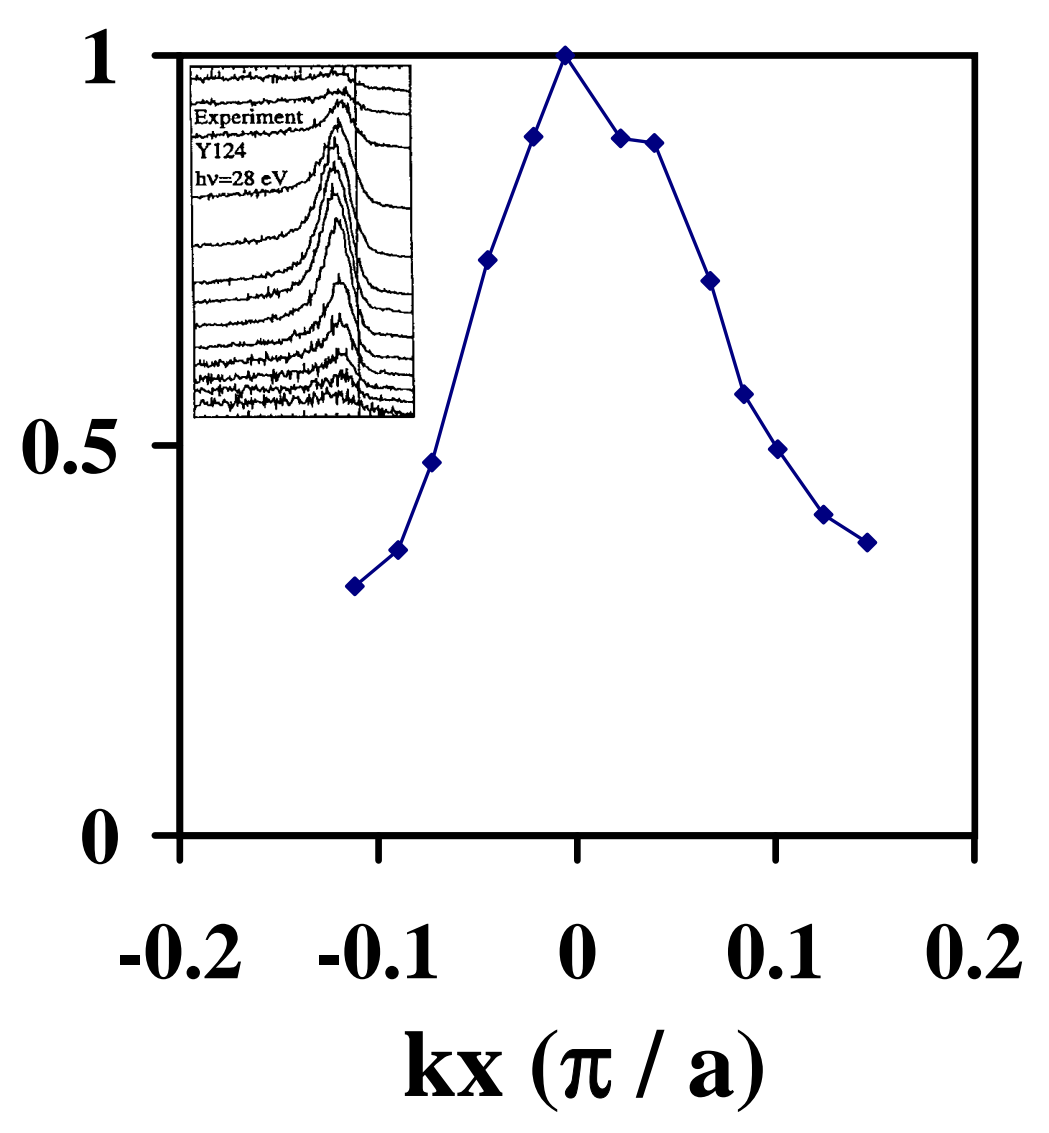


(a)

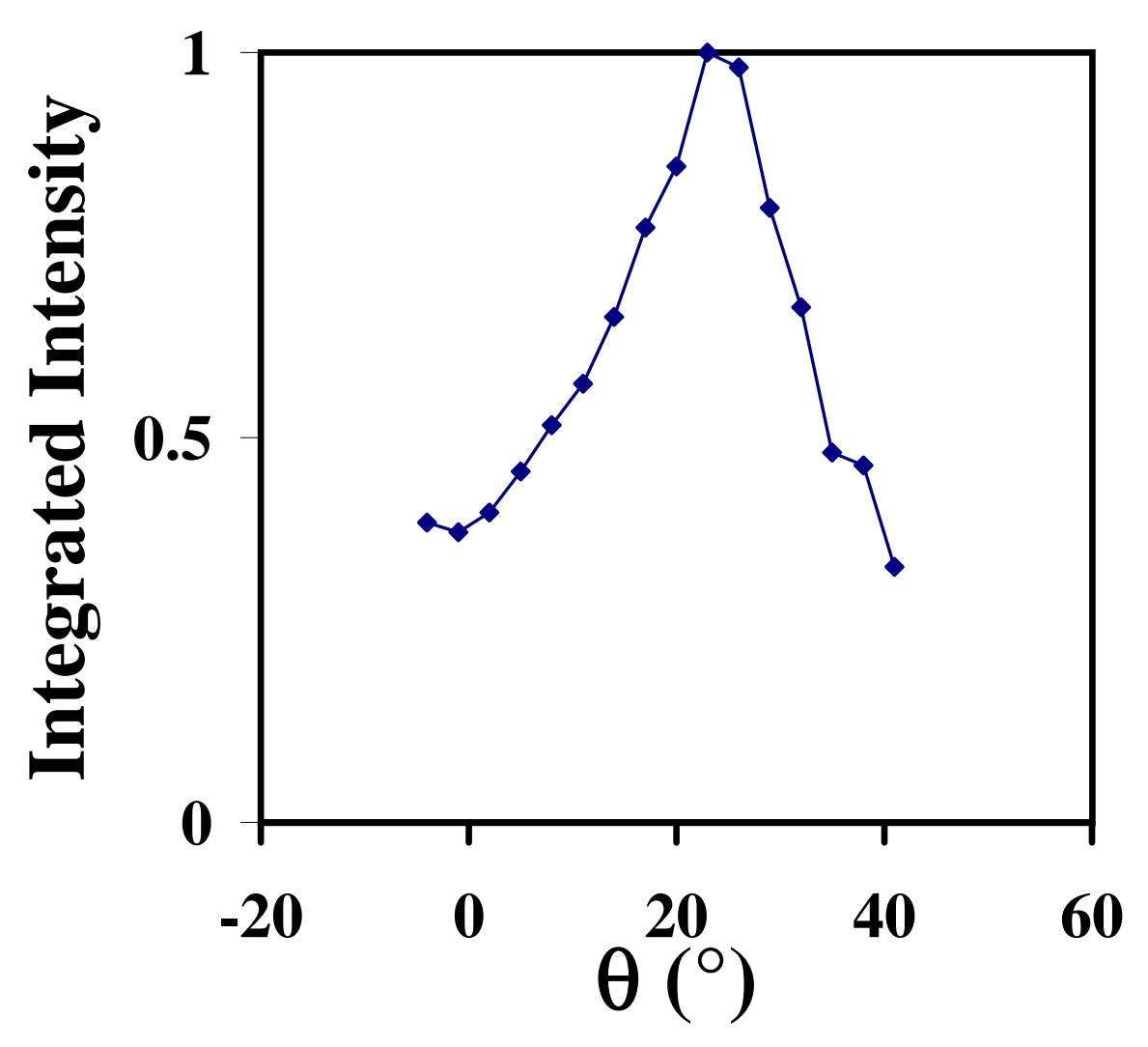

(b)

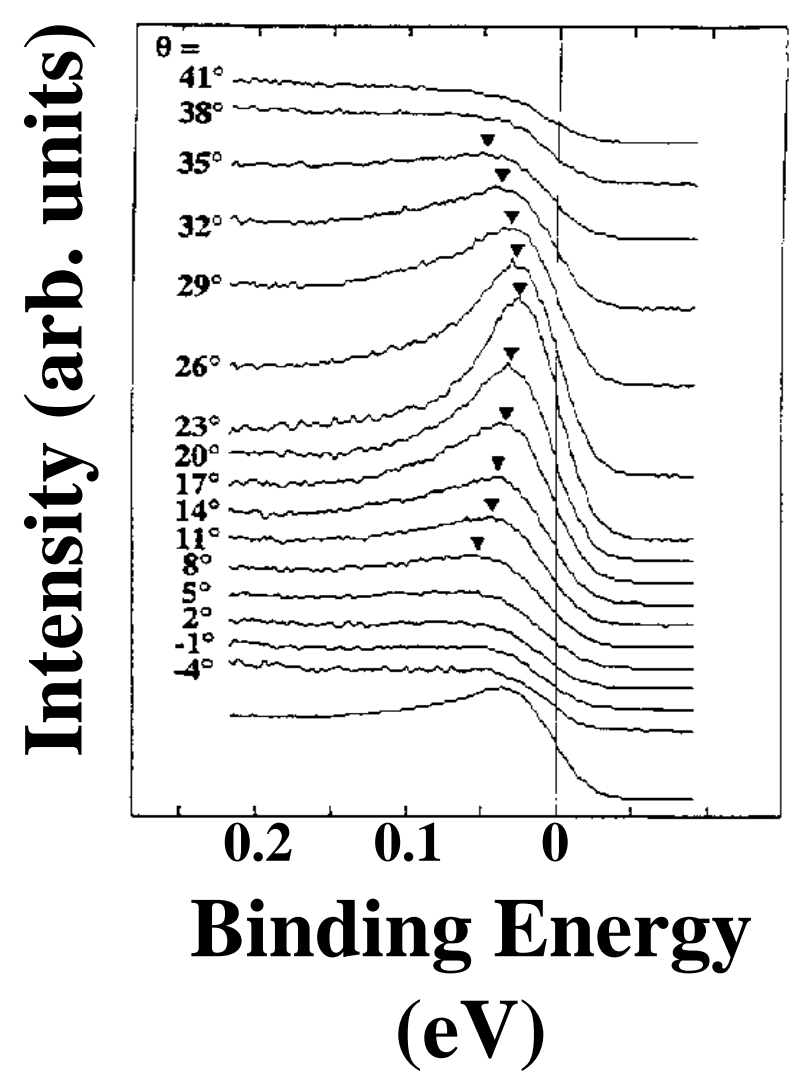




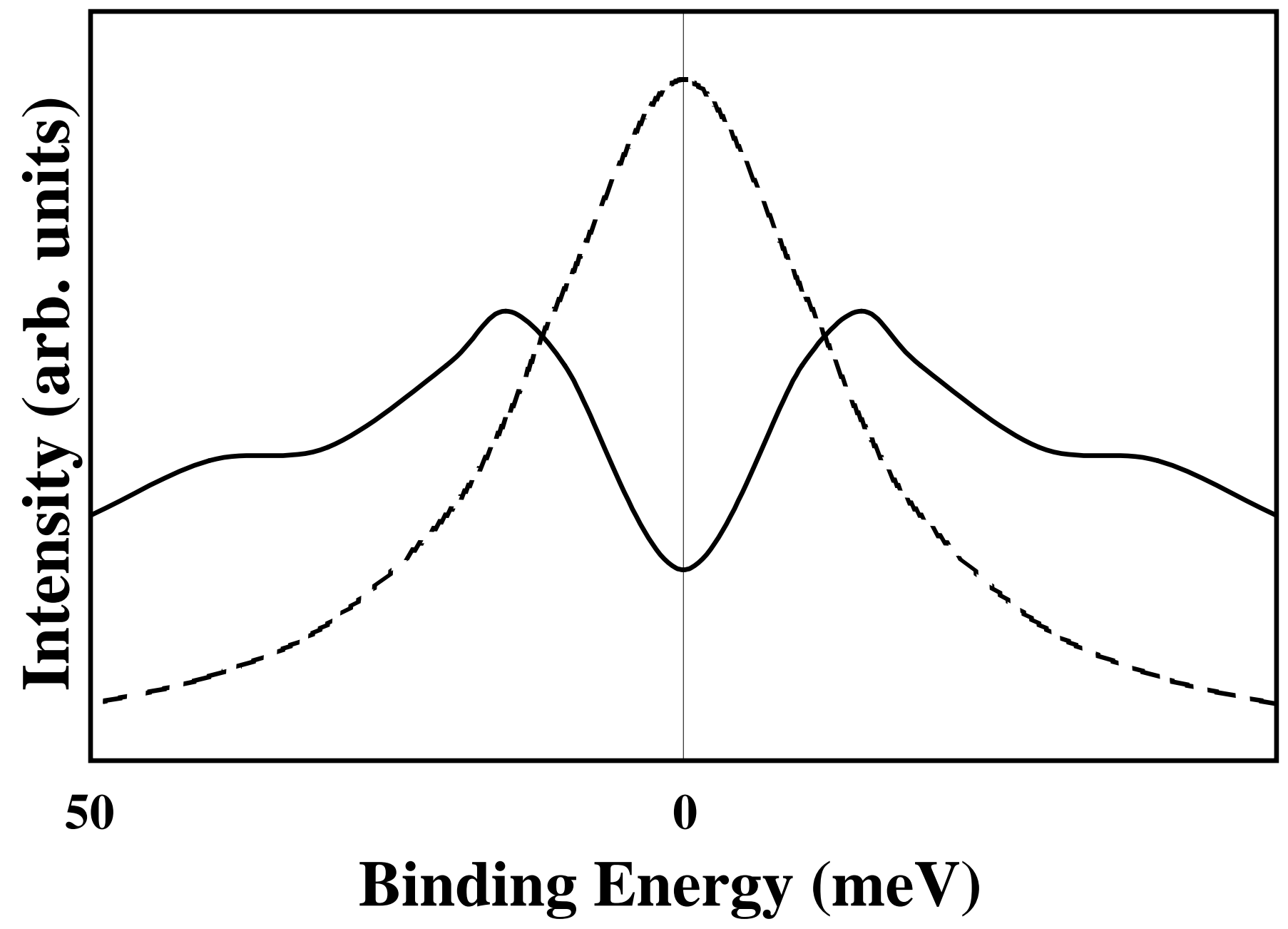

